\title{
The concept of anti-collision system of autonomous surface vehicle
}

\author{
Andrzej Stateczny ${ }^{1}$, and Waldemar Gierski ${ }^{1, *}$ \\ ${ }^{1}$ Gdansk University of Technology, Faculty of Civil and Environmental Engineering, Narutowicza St. 11/12, Gdansk, Poland
}

\begin{abstract}
The use of unmanned vehicles in various fields of science and the economy is becoming more common. An extremely important aspect of creating this type of solution is to provide autonomous vehicle navigation, which does not require interference of the human factor or in which it is limited to a minimum. This article discusses the concept of autonomous anti-collision system of unmanned surface vehicle. It proposed a sensor system for the comprehensive implementation of the vehicle path in an automatic manner and presented the idea of remote sensing data fusion.
\end{abstract}

\section{Introduction}

The use of unmanned vehicles (UVs) in various fields of science and life is becoming more and more common. Although previously used mainly for military and cosmic purposes, the willingness to study inaccessible places of the earth, gather information about disasters and occurrences at areas of immediate danger to people and facilitate research missions, these experiences were transferred to other applications. The advantages of autonomous vehicles allow their use in scientific research and in the amenities of everyday life. The use of unmanned vehicles for exploratory purposes allows us to work using the smaller human resources involved in measuring service, which makes it more flexible. This saves time and reduces the human factor that can significantly affect the quality of measurements. UVs are characterized by smaller dimensions and weight, which makes it possible to carry out more detailed tests (possible measurement in places where the vehicle on which the people are located could not perform them). Research UVs, as well as those used in everyday life (e.g. for passenger transport) perform their tasks more accurately, their efficiency and attention level does not deteriorate as a result of long working time and fatigue. An additional advantage is low energy consumption, and thus low labor costs. An extremely important aspect of using autonomous UVs for different tasks is detecting obstacles standing in the way of the vehicle in real time. One of the most difficult elements of the concept of an unmanned vehicle is designing the anti-collision system and developing a method of data fusion from various sensors detecting obstacles on a programmed path in order to obtain their reliable avoidance for the safe exploitation of the unit.

The construction of a surface vehicle and development of a sensor system concept requires predicting the conditions under which it will be operated and assumptions in recognizing objects and modelling the environment in which the autonomous surface vehicle (ASV) will move. ASVs are used in many scientific and commercial realizations. They are used by the army for reconnaissance and combat purposes, they serve as research units providing information on various aspects of the aquatic environment or carriers of measuring equipment for the inventory of watercourses and reservoirs. Most of the current solutions work on the basis of remote control. The operator, using the control module, realizes movement of the unit from a certain distance, based on a visual assessment of the surrounding. However, current trends in the development of this type of solutions seek to marginalize human participation in the implementation of the unmanned vehicle path. The main problems in the development of the autonomous navigation system are specific working conditions (contact of two environments with completely different physical characteristics: water - air), difficulty in imitating human senses in navigation with appropriate algorithms or inference structures, as well as in the case of coastal areas, the boundary between water and land. Although the selection of sensors available on the market gives a wide possibility of detecting objects on ASV path, prosaic factors such as the dimensions and weight of the unit are a significant limitation. This restricts the system's recording module, therefore it is timeconsuming to develop a design that will ensure optimal detection with the lowest possible usage of batteries. The complexity of environmental conditions and design constraints affects the selection of measuring equipment. The order in which sensors are first selected causes that data fusion and the choice of path implementation methods is an extremely complicated task. All this causes that the development of an autonomous anticollision system in the case of ASV is a very growing and innovative subject.

\footnotetext{
* Corresponding author: waldemar.gierski@pg.edu.pl
} 


\section{The structure of the anti-collision system}

Autonomous anti-collision systems in unmanned vehicles are based on the registration of many sensors. The combination of data from all of them and their proper processing provide an optimal solution for the obstacle-free path of the surface platform. The programmed route (global path) of the vehicle must be supplemented in real time with static obstacles appearing on its way and those in motion relative to unmanned vehicle. In addition, the task of the automatic navigation system is to determine and implement the corrected path. Each of the sensors used to detect obstacles has a number of limitations related to range, detection resolution and optimal working conditions. Proper selection of sensors allows mutual complementation of the obtained information and thus as accurately as possible to determine the parameters of the obstacles necessary to avoid them in the safe way. The structure of the proposed navigation system that can be used in a ASV is given below, taking into account the small dimensions of the vehicle, the conditions of operation and the mutual complementation of data in the navigation process $[1,2]$.

\subsection{GNSS receiver and global path planning}

Navigation elements using GNSS sensors are the basic assumption of most collision avoidance systems. For proper operation of any sensor it is necessary to determine the ASV exact position in the assumed reference system and to implement detection of objects in its surrounding (in the same reference system). In this case, the GNSS positioning systems are the simplest in application, they give accurate data and enable the simplest interoperability of the anti-collision system with navigational charts and digital terrain models [3]. The GNSS receiver installed on the surface platform enables the implementation of the global path of the vehicle, i.e. the course assumed to be overcome during a specific vehicle mission [4].

Nowadays, GNSS receivers are readily available and there is a huge selection of these sensors in terms of parameters necessary in a given anti-collision system. The one of solution is Trimble $\mathrm{R} 10$. The receiver has a small size $(11.9 \times 13.6 \mathrm{~cm})$ and weight $(1.12 \mathrm{~kg})$ when carrying out the measurement will very high accuracy and resolution, sufficient to determine the position in the tracking of the vehicle. It allows measurement with horizontal accuracy: $8 \mathrm{~mm}+0.5 \mathrm{ppm}$ RMS and vertical accuracy: $15 \mathrm{~mm}+0.5 \mathrm{ppm}$ RMS in real time measurement mode with network transfer of corrections. It has a function of wireless transmission of registration data and is characterized by a low energy consumption of $5.1 \mathrm{~W}$, which argues its use in the discussed system. Tracking satellite signals from more than one satellite system (GPS, GLONASS) affects the reliability of measurement.

Due to the fact that the ASV can be used for measurements in places where terrain apertures may interfere with signal tracking and prevent precise position determination of the antenna, it is proposed to supplement the system. In the case of vehicle operation in a small area (at the shore), the supplement may be a prism system arranged on the deck, whereby it would be possible to determine the position by continuous measurement from the shore of the basin using a total station supported by its own GNSS receiver. In the case of carrying out tasks of the unit in open waters, the solution may be inertial navigation performed by IMU sensors (gyroscope, accelerometer).

An idea of using the IMU sensors is very promising. The second solution of navigation data source is SBG Ekinx-2U. Integrated motion unit with GPS RTK receiver is especially useful during performing hydrographical tasks. Important parameters for anticollision tasks like position and speed vector accuracy is comparable with Trimble R10 and exceed navigational conditions.

\subsection{RADAR sensor}

In the case of ASVs, it is very important to detect coastline and keep away from it at the distance of safe maneuvering of the vehicle. The optimal solution for tracking the surrounding of small unmanned platforms is the automotive Doppler RADAR. The principle of operation is the phenomenon of Doppler shift caused by the movement of objects from which the emitted beam of electromagnetic radiation is reflected. When receiving a beam in RADAR, depending on the type of RADAR, the intensity of the received beam is analyzed, the time of return of the beam, and in Doppler's, its frequency, on the basis of which the speed of the moving object is determined. RADAR is used not only to detect objects and determine their position, but also the direction (getting closer, getting away) and speed of getting closer/ getting away (Fig.1. shows the visualization of measurements using RADAR).

The advantages of using RADAR in detecting obstacles are [5]:

- Long detecting range,

- Good velocity estimates,

- Provides nearly all-weather and broad-area imagery,

- High depth resolution and accuracy.

The limitations are:

- Skewed data from fast turning maneuvers depending on the context,

- Limited small and dynamic target detection capability,

- Susceptible to high waves and water reflectivity.

The proposed device for RADAR tracking objects disturbing the path of the vehicle is UMRR Type 42 mobile RADAR. It is characterized by a range of 1.5 to $340 \mathrm{~m}$, a distance measurement accuracy of $0.25 \mathrm{~m}$ and a field of view of horizontally: $100^{\circ}$ and vertically: $16^{\circ}$. Reflectors having a radial speed component of typ. abs. $>0.1 \mathrm{~m} / \mathrm{s}$ are detected. Having multi target capability, the sensor may detect many reflectors at a time (up to 256) being within the field of view. Depending on the selected communication interface, the number of reported targets may be limited to 128 . Targets are sorted by range and if 
more than 128 are detected, short range targets are reported first. Dimensions of the sensor are around: 230 x $160 \mathrm{~mm}$, a weight: $1290 \mathrm{~g}$. Energy consumption is relatively low while maintaining high accuracy of measurements and amounts to $10.5 \mathrm{~W}$ [6].

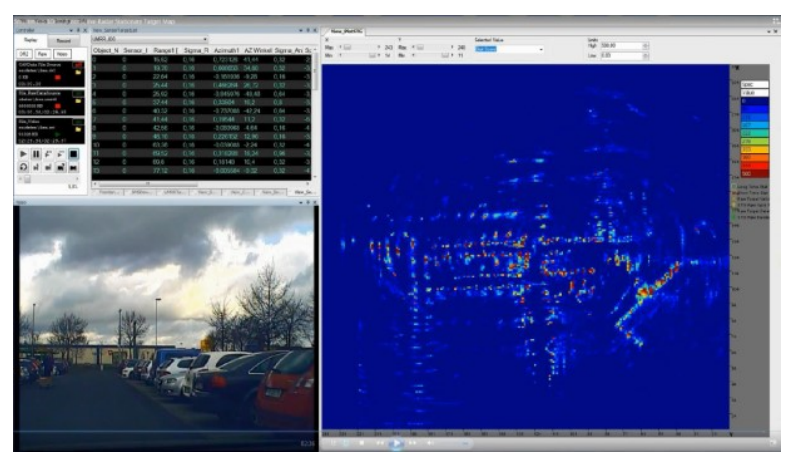

Fig. 1. Stationary target GRID visualization from the automotive RADAR sensor called UMRR Type 42 Antenna $[6]$.

Appropriate installation of the sensor on the ASV, where the construction elements do not limit the field of view, gives very high quality navigation information, which makes the RADAR the core of the anti-collision system. The importance of using RADAR in this type of vehicles is evidenced by the widespread use of RADARs on ships. RADAR images are particularly helpful when driving a vehicle in an offshore environment or intensively developed areas of hydrotechnical infrastructure (e.g. in port, roads, wharves areas).

\subsection{LiDAR sensor}

The use of LiDAR sensors in autonomous navigation (anti-collision systems) of unmanned vehicles is associated with their high accuracy, speed and range. Data from the system give a continuous, spatial representation of the surface area around the vehicle, while allowing to determine the velocity of movement and the path of movement in relation to the characteristic elements of the surrounding. A laser rangefinder, sent through a scanning mirror or fiber optic system, scans the area in a plane transverse to the direction of motion. The laser is pulsed and samples the terrain with great frequency, the energy partially reflected from the terrain surface or the obstacles is picked up by the optical system of the scanner and recorded. Based on the measurement of the return time of the reflected signal also allows you to determine the physical features and structure of the object from which the impulse returned to the device. An accurate representation of the environment is required to avoid obstacles. LiDAR data allows orientation of objects and vehicle in all dimensions (3D) of the adopted coordinate system. It gives greater possibilities of anti-collision system. The use of LiDAR in real-time realizations is a difficult task, but with the use of artificial intelligence elements it allows a very accurate visualization of the situation around the vehicle and is extremely helpful in the navigation of unmanned vehicles.
The data from the LiDAR measurement are in the form of a three-dimensional and $360^{\circ}$ cloud of points (Fig. 2) for which the coordinates $\mathrm{x}, \mathrm{y}, \mathrm{z}$ in the assumed coordinate system, intensity and beam number (ring number) have been determined. The $\mathrm{x}, \mathrm{y}, \mathrm{z}$ coordinates represent the position of each point relative to signal source. Intensity represents the strength of the signal returning to the receiver (depends on the reflective properties of the object from which the wave was reflected). The beam number determines in which beam the returned point is located [7]. The main advantages of this method of acquiring data about the surrounding are [5]:

- Good at near range obstacle detection,

- High depth resolution and accuracy.

The limitations are:

- Angular resolution both vertically and horizontally,

- Sensitive to environment and ASV motion.

An exemplary sensor that can be used in ASVs is 3D real-time Velodyne VLP-16 LiDAR. Performs measurements in the range of full angle in the horizontal plane and $30^{\circ}$ in the vertical plane. It is characterized by compact dimensions and weight (830 g) and low power consumption which additionally supports the legitimacy of its use on small vehicles. Small size retains high accuracy of the registration of the surrounding (16 channels, $100 \mathrm{~m}$ range, up to 600000 points per second). Fig. 2 shows the visualization of measurements using said LiDAR. When implementing the design of the unit, it should be remember that LiDAR, like RADAR, should be located in such a way that other elements of the platform construction do not interfere with the registration area [8].

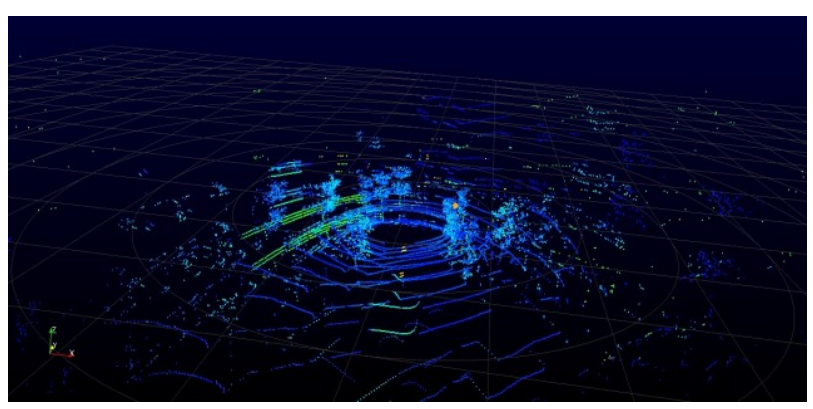

Fig. 2. Visualization of the point cloud from the mobile LiDAR sensor Velodyne VLP-16 [8].

\subsection{Visual sensors}

Visual methods are used to detect obstacles due to the simplicity of their use, relatively high efficiency and low installation costs. Stereo vision-based anti-collision systems detect objects that may interfere with the movement of the vehicle on its implemented path. They enable differentiation of objects due to their shape, color and texture, the type of the object and its localization are determined.

Detecting obstacles on stereo images is complicated. It is not know in advance how many objects will be on the each of the input images, it is also not known where in the image they will appear or what dimensions they 
will have. The problem is quite difficult to solve, hence the method of using artificial neural networks is often used to decode the images into information for the anticollision system [7].

The advantages of vision sensors are:

- High lateral and temporal resolution,

- Simplicity and low weight in practical application.

The limitations are:

- Low depth resolution and accuracy,

- Challenge to real-time implementation,

- Susceptible to light and weather conditions.

Visual sensors have been selected for the ability to supplement laser (LiDAR) and radar measurements to increase system reliability. Analysis of the RGB stereo camera recording enables noise isolation and exclusion of electromagnetic and laser reflections that are not relevant to the vehicle path. It allows to increase the range of observation and to indicate to other sensors approximate directions and distances to potential obstacles. Interpretation of visual registrations is based on the geometrical relations of the image (the distance, angle and confidence score from the camera are used to create a new direction). The placement and number of sensors on the platform depends on the technological assumptions of its operation. Cameras should be chosen so as to realize the visualization of the surroundings, especially in the direction of vehicle movement.

The installation of a metric cameras system would be the best solution in this type of application of vision methods. However, the complexity and sensitivity of optical systems, large dimensions and weight, as well as high costs limit their use in unmanned units, where space for assembly is limited, and energy consumption and lightweight design prevent their assembly. The solution is widely used, e.g. in unmanned aerial vehicles, nonmetric cameras. After the self-calibration process, based on geometrical relationships, they are good source for obtaining relatively accurate photogrammetric data, sufficient for the navigation process [9].

An example of a camera that meets the assumptions of the anti-collision system is DJI ZENMUSE X5S 4K DJI Inspire 2. It enables image recording up to $60 \mathrm{fps}$ in $1920 \times 1080$ pixels resolution ( $30 \mathrm{fps}$ in $3840 \times 2160$ ). It is a 20.8-megapixel camera with a $4 / 3$ " CMOS sensor. It works in the temperature range: -10 to $+40^{\circ} \mathrm{C}$. It is characterized by reliable image stabilization in the area of: pitch: $-90^{\circ}$ to $+30^{\circ}$, pan: $\pm 320^{\circ}[10]$. These cameras are commonly used in advanced unmanned measuring aircrafts, so it is ideally suited for a floating platform. The development of the camera deployment concept depends on the intended use of the ASV.

\subsection{Data acquisition and processing module}

The proposed sensors have proprietary software for analyzing and developing the results of their measurements. The data processing system of the autonomous navigation system of the unmanned platform connects these separate applications together, co-creating a uniform result of the vehicle's environment representation. As the remote sensing sensors proposed are intended for mobile applications, the results of their work are sent to the developed analyzer and combined into one coherent information about the location of obstacles in the way of the vehicle.

Data for each sensor should be processed independently and after processing should be fused to establish common collision situation image. Main task for processing algorithms is to detect moving and fixed target. Shore constructions and other nonmoving targets not need target tracking procedure. Anti-collision algorithms for nonmoving targets provide simply rules not to hit fixed constructions.

For moving targets anti-collision system needs target tracking algorithms. According to previous research the best way is to use neural network tracking filter. Especially useful appears filter based on General Regression Neural Networks. Patented filter consist of two working parallelly General Regression Neural Networks was presented in previous works [11-14].

After detecting of nonmoving targets and moving target tracking procedure data from different sensors should be fused. An idea of different sensors fusion was presented in [15].

Some authors analyzed the various aspects of unmanned vehicles navigation. Video sensor and LiDAR fusion are described in [16]. In [17], an algorithm using LiDAR and a camera for detecting and tracking surface obstacles using the Kalman filter is presented. Legal aspects of ASV navigation, including anti-collision, are described in [18]. An approach using artificial neural networks (ANN) to solve the ASV anti-collision problem is presented in [19-21], where ANN was used to control the autonomous robot. 3D mobile (3D LiDAR ) and GNSS applied to autonomous car navigation was presented in [22].

\section{Automatic implementation of the path improved by data from the anti-collision system}

For the implementation of the unmanned vehicle path complex algorithms are used which, based on the data registered by the sensors, determine the route on which no collision with another object will occur. For this purpose, analyses of fused data collected by the anticollision system or intelligent methods are used.

The future of this type of systems is marked by the use of artificial intelligence (AI) methods. AI is used in engineering practice to solve problems in the absence of all information necessary for their analytical solution. Simulation of human brain function, the ability to learn from previous system experience, the ability to make decisions in situation where IT systems can not solve the problem are just a few advantages of using AI in the problem of autonomous navigation of unmanned vehicles. Among the many practical applications of AI, artificial neural networks (ANN) are particularly interesting in the process of automatic implementation of the path by the vehicle. The operation of the autonomous anti-collision system, in which activities are undertaken by ANN, depends on the way the network is taught. 
Learning is accomplished by the task of conditions according to which the ANN will be able to solve the set task. The effectiveness of ANN depends on the learning phase, the method of action should be properly determined depending on the input signals, the scales of connections neurons responsible for specific tasks and threshold values of neurons should be properly resolved. Correct defining of the network's assumptions allows for the implementation of a specific purpose.

It is also possible to use intelligent algorithms based on fuzzy logic and evolutionary algorithms. Interesting approach of intelligent path planning using evolutionary strategy was presented in [23-25].

In future authors works artificial intelligence method will be tested to provide optimal maneuvering solution for ASV. The most important idea for autonomous vehicle navigation is own ship safety.

\section{Summary}

Combined analysis of data acquired using various sensors, in this case, LiDAR, RADAR and video sensors, by sensor fusion of navigation sensors will allow to obtain a synergic effect, useful in the aspect of anti-collision maneuvering of future oriented fully autonomous intelligent unmanned vehicles.

The new idea, proposed in the article, not known in available worldwide bibliography, is multisensory fusion set of lidars, radars and cameras for establish intelligent fully autonomous navigation system for ASV based not only on one source of data like it is provided for now but based on fused multisensory data: from those sensors.

\section{References}

1. R. Polvara, S. Sharma, J. Wan, A. Manning, R. Sutton, Obstacle avoidance approaches for autonomous navigation of unmanned surface vehicles, The Journal of Navigation, 71 (2017)

2. A. Romano, P. Duranti, Autonomous unmanned surface vessels for hydrographic measurement and environmental monitoring, FIG Working Week, Rome, (2012)

3. Y. Aotani, T. Ienaga, N. Machinaka, Y. Sadakuni, R. Yamazaki, Y. Hosoda, R. Sawahashi Y. Kuroda, Development of autonomous navigation system using $3 D$ map with geometric and semantic information, Journal of Robotics and Mechatronics, 29, 4 (2017)

4. X. Meng, H. Wang, B. Liu, A robust vehicle localization approach based on GNSS/IMU/ DMI/ LiDAR sensor fusion for autonomous vehicles, Sensors, 17, 9 (2017)

5. Z. Liu, Y. Zhang, X. Yu, C. Yuan, Unmanned surface vehicles: an overview of developments and challenges, Annual Reviews in Control, 41 ( 2016)

6. UMRR Type 42 Antenna Data Sheet

7. P. Wei, L. Cagle, T. Reza, J. Ball, J. Gafford, LiDAR and camera detection fusion in a real-time industrial multi-sensor collision avoidance system, Electronics 7, 6 (2018)

8. Velodyne VLP-16 Sample Data

9. C.-H. Tsai, Y.-C. Lin, An accelerated image matching technique for UAV orthoimage registration, ISPRS Journal of Photogrammetry and Remote Sensing, 128 (2017)

10. ZENMUSE X5S Specification

11. A. Stateczny, Neural manoeuvre detection of the tracked target in ARPA systems, Proceedings CAMS 2001, Glasgow, (2001)

12. A. Stateczny, W. Kazimierski, Selection of GRNN network parameters for the needs of state vector estimation of manoeuvring target in ARPA devices, Photonics Applications in Astronomy, Communications, Industry, and High-Energy Physics Experiments IV, Wilga, (2005)

13. W. Kazimierski and A. Stateczny, Optimization of multiple model neural tracking filter for marine targets, 13th International Radar Symposium (IRS), Warsaw, (2012)

14. W. Kazimierski, G. Zaniewicz, A. Stateczny, Verification of multiple model neural tracking filter with ship's radar, 13th International Radar Symposium (IRS), Warsaw, (2012)

15. W. Kazimierski, A. Stateczny, Fusion of data from AIS and tracking radar for the needs of ECDIS, Signal Processing Symposium Jachranka, Poland, (2013)

16. J. Jo, Y. Tsunoda, B. Stantic et al., A likelihoodbased data fusion model for the integration of multiple sensor data: a case study with vision and Lidar sensors, Robot Intelligence Technology And Applications 4, 447, (2017)

17. L. Jooho, W. Joohyun W., K. Nakwan, Obstacle avoidance and target search of an autonomous surface vehicle for 2016 maritime RobotX Challenge, IEEE OES International Symposium on Underwater Technology (UT), Busan, (2017)

18. J. H. Mei, M. R. Arshad, COLREGs based navigation of riverine autonomous surface vehicle, IEEE 6TH International Conference on Underwater System Technology, Malaysia, 2016

19. A. Barton, E. Volna, control of autonomous robot using neural networks, Proceedings of the International Conference on Numerical Analysis and Applied Mathematics 2016 (ICNAAM-2016), Rhodes, 2016

20. B. Ko, H.J. Choi, C. Hong et al., Neural networkbased autonomous navigation for a homecare mobile mobot, 2017 IEEE International Conference On Big Data And Smart Computing (BIGCOMP), Jeju 2017

21. T. Praczyk, Neural anti-collision system for autonomous surface vehicle, Neurocomputing, 149 (2015)

22. J. Lil, H. Bao, X. Han et al., Real-time self-driving car navigation and obstacle avoidance using mobile 
$3 D$ laser scanner and GNSS, Multimedia Tools and Applications, 76 (2016)

23. P. Kolendo, R. Smierzchalski,, Ship evolutionary trajectory planning method with application of polynomial interpolation, Activities in Navigation: Marine Navigation and Safety of Sea Transportation (2015)

24. L. Kuczkowski, R. Smierzchalski, Termination functions for evolutionary path planning algorithm 19th International Conference on Methods and Models in Automation and Robotics (MMAR) Miedzyzdroje, (2014)

25. L. Kuczkowski, R. Smierzchalski, Comparison of single and multi-population evolutionary algorithm for path planning in navigation situation, Symposium on Mechatronics Systems, Mechanics and Materials, Jastrzebia Gora, (2013) 